\title{
Modeling Rocket Flight in the Low-Friction Approximation
}

Logan White

University of South Florida

\author{
Advisors: \\ Manoug Manougian, Mathematics and Statistics \\ Razvan Teodorescu, Physics \\ Problem Suggested By: Razvan Teodorescu
}

Follow this and additional works at: https://digitalcommons.usf.edu/ujmm

Part of the Mathematics Commons

UJMM is an open access journal, free to authors and readers, and relies on your support:

Donate Now

\section{Recommended Citation}

White, Logan (2014) "Modeling Rocket Flight in the Low-Friction Approximation," Undergraduate Journal of Mathematical Modeling: One + Two: Vol. 6: Iss. 1, Article 5.

DOI: http://dx.doi.org/10.5038/2326-3652.6.1.4861

Available at: https://digitalcommons.usf.edu/ujmm/vol6/iss $1 / 5$ 


\title{
Modeling Rocket Flight in the Low-Friction Approximation
}

\begin{abstract}
In a realistic model for rocket dynamics, in the presence of atmospheric drag and altitude-dependent gravity, the exact kinematic equation cannot be integrated in closed form; even when neglecting friction, the exact solution is a combination of elliptic functions of Jacobi type, which are not easy to use in a computational sense. This project provides a precise analysis of the various terms in the full equation (such as gravity, drag, and exhaust momentum), and the numerical ranges for which various approximations are accurate to within $1 \%$. The analysis leads to optimal approximations expressed through elementary functions, which can be implemented for efficient flight prediction on simple computational devices, such as smartphone applications.
\end{abstract}

\section{Keywords}

Differential Equations, Rocket Flight, Motion 


\section{PROBLEM STATEMENT \& MOTIVATION}

The question under investigation in this paper is: How can we best model rocket flight with closed-form equations?

\section{MATHEMATICAL DESCRIPTION AND SOLUTION APPROACH}

\section{EXACT SOLUTIONS}

Assuming that the relationship between the mass of the rocket $m(t)$ at time $t$ and the rate of mass depletion $m^{\prime}(t)$ is proportional, gives

$$
\frac{d m}{d t}=-Q m(t)
$$

for some constant $Q$. Hence the mass remaining at time $t$, found through separation of variables and subsequent integration, is

$$
m(t)=m_{0} e^{-Q t} \text { where } t \geq 0
$$

and $m_{0}=m(0)$ is the initial mass of the rocket. By Newton's Second Law, the sum of forces $F_{i}$ on an object equals the product of the object's mass and acceleration:

$$
\sum_{i=1}^{n} F_{i}=m \frac{d v}{d t}
$$

The forces summed in the direction of the rocket's flight following liftoff are the gravitational force: 


$$
F_{g}=-\frac{G m_{e} m(t)}{y^{2}}
$$

where $G$ is Newton's gravitational constant, $m_{e}$ is Earth's mass, and $y=y(t)$ is the vertical position of the rocket relative to Earth's center, and the force of thrust caused by the ejection of fuel out of the rocket's nozzle:

$$
F_{t}=-c \frac{d m}{d t}
$$

where $c$ is the constant exhaust speed, relative to the rocket.

Letting $\alpha=G m_{e}$ and $\beta=Q c$ and combining equations (2), (3), and (4), we receive a differential equation into which we can substitute equation (1) like so:

$$
m(t) \frac{d v}{d t}=-\frac{\alpha m(t)}{y^{2}}-c \frac{d m}{d t} \Rightarrow\left(m_{0} e^{-Q t}\right) \frac{d v}{d t}=-\frac{\alpha m_{0} e^{-Q t}}{y^{2}}+\beta\left(m_{0} e^{-Q t}\right)
$$

Simplifying, we receive

$$
\frac{d v}{d t}=\beta-\frac{\alpha}{y^{2}}
$$

Noticing that $v=d y / d t$ :

$$
\frac{d v}{d t}=\frac{d v}{d y} \frac{d y}{d t}=v \frac{d v}{d y}
$$

Substituting equation (6) into our second-order differential equation (5), we arrive at the equation:

$$
v \frac{d v}{d y}=\beta-\frac{\alpha}{y^{2}} \quad \Rightarrow \quad v d v=\beta d y-\frac{\alpha}{y^{2}} d y
$$

and integrate: 


$$
\int_{v=0}^{v(t)} v d v=\int_{y=r_{e}}^{y(t)} \beta d y-\int_{y=r_{e}}^{y(t)} \frac{\alpha}{y^{2}} d y \Rightarrow \frac{[v(t)]^{2}}{2}=\beta\left[y(t)-r_{e}\right]-\frac{\alpha\left[y(t)-r_{e}\right]}{r_{e} y(t)},
$$

noting that the rocket begins its flight on the Earth's surface, i.e., $y(0)=r_{e}$ where $r_{e}$ is the radius of the Earth. If we substitute $v(t)=d y / d t$, we arrive at

$$
\left(\frac{d y}{d t}\right)^{2}=2 \beta\left[y-r_{e}\right]-2 \frac{\alpha\left[y-r_{e}\right]}{r_{e} y}
$$

Separating variables in equation (7) gives

$$
d t=\frac{d y}{\sqrt{2 \beta\left[y-r_{e}\right]-2 \frac{\alpha\left[y-r_{e}\right]}{r_{e} y}}}
$$

and integrating yields

$$
\int_{t=0}^{t} d t=\int_{y=r_{e}}^{y(t)} \frac{d y}{\sqrt{2\left[y-r_{e}\right]\left[\beta-\frac{\alpha}{r_{e} y}\right]}}=\int_{z=0}^{z(t)} \frac{d z}{\sqrt{\frac{2 \alpha}{r_{e}[z]\left[\frac{r_{e} \beta}{\alpha}+\frac{-\frac{1}{r_{e}}}{1-\left(-\frac{z}{r_{e}}\right)}\right]}}}
$$

and we get an equation that is difficult to use. This solution is not practical from a computational standpoint, as it involves two different types of Jacobi elliptic integrals. Instead, it would probably be more useful to investigate various methods of approximation by which we can simplify the function further.

\section{APPROXIMATIONS AND ERROR}

The simplest approximation of equation (8) that we consider neglects the effect of the $\frac{-\frac{1}{r_{e}}}{1-\left(-\frac{z(t)}{r_{e}}\right)}$ term. This approximation leads to a solution that is unsatisfactory because it ignores the effects of gravity: 


$$
z(t)=\frac{\beta}{2} t^{2}
$$

In order to arrive at a more satisfactory solution, we will have to look at geometric series. The sum of the infinite and convergent geometric series $\sum_{i=1}^{\infty} a_{i}$ is $\frac{a_{1}}{1-r}$, where $a_{1}$ is the first term of the series and $r$ is the common ratio. The term in the denominator of the right side of equation (8) that makes the equation difficult to integrate is

$$
\frac{-\frac{1}{r_{e}}}{1-\left(\frac{-z(t)}{r_{e}}\right)}=-\frac{1}{r_{e}}+\frac{z(t)}{r_{e}^{2}}-\frac{[z(t)]^{2}}{r_{e}^{3}}+\cdots
$$

when expressed as the sum of an infinite geometric series with $a_{1}=-\frac{1}{r_{e}}$ and $r=-\frac{z(t)}{r_{e}}$

The total sum (10) can be approximated by simply taking the first few terms of the series. All of this can be done only under the assumption that $z(t) \ll r_{e}$, which makes the series convergent. However, this is always going to be true for the first stage of any multi-stage rocket flight. The error of any order approximation is going to be less than $0.01(=1 \%)$ as follows:

$$
S_{n}=-\frac{1}{r_{e}}+\frac{z(t)}{r_{e}^{2}}-\frac{[z(t)]^{2}}{r_{e}^{3}}+\cdots+a_{n}+\cdots
$$

$a_{n}$ is neglected as having less than $1 \%$ of the total sum $S_{n}$. So

$$
S_{n}=-\frac{1}{r_{e}}+\frac{z(t)}{r_{e}^{2}}-\frac{[z(t)]^{2}}{r_{e}^{3}}+\cdots+R_{n}
$$

where $R_{n}$ is the remainder and error term used to represent the terms $a_{n}$ and beyond. In particular, 


$$
R_{n}=\frac{a_{n}}{1-\left(-\frac{z(t)}{r_{e}}\right)}
$$

Because $a_{n}<0.01$, it must be true that $R_{n}<0.01$, so all errors are less than $1 \%$.

\section{i. ZERO-ORDER APPROXIMATION}

In the zero-order approximation, the first term is taken from the infinite series (10) and used to approximate the sum of the series. When this substitution is carried out and the integrand simplified, we obtain

$$
t=\int_{z=0}^{z(t)} \frac{d z}{\sqrt{2 z(t)(\beta-g)}}=\frac{1}{2(\beta-g)} \sqrt{2 z(t)(\beta-g)}
$$

Solving for $z(t)$, we get

$$
z(t)=\frac{t^{2}}{2}(\beta-g)
$$

If the zero-order approximation is only reasonable when $\frac{z(t)}{r_{e}} \leq 0.01$, the time domain $0 \leq t \leq T_{0}$ can be found as follows:

$$
0.01 r_{e}=T_{0}^{2}\left(\frac{\beta-g}{2}\right) \Rightarrow T_{0}=\sqrt{\frac{0.02 r_{e}}{\beta-g}} .
$$

Equation (11) provides a quadratic approximation of the first stage of a rocket flight; however, it does not account for variation in gravitational force. Instead, it assumes a constant force $m g$ that would only be present at Earth's surface. Because we are assuming a varying gravitational force, it is necessary to use a first-order approximation.

\section{ii. FIRST-ORDER APPROXIMATION}


Taking the first two terms of the series (10) and substituting in for the sum of the series, we arrive at

$$
\int_{t=0}^{t} d t=\int_{z=0}^{z(t)} \frac{d z}{\sqrt{\frac{2 \alpha}{r_{e}}(z)\left(\frac{r_{e} \beta}{\alpha}-\frac{1}{r_{e}}+\frac{z}{r_{e}^{2}}\right)}}=\int_{z=0}^{z(t)} \frac{d z}{\sqrt{A\left[(z+\kappa)^{2}-\kappa^{2}\right]}}
$$

where $A=\frac{2 g}{r_{e}}$ and $\kappa=\frac{\beta-g}{A}$. Using the trigonometric substitution $z=\kappa \sec \theta-\kappa$ and $d z=$ $\kappa \sec \theta \tan \theta d \theta$, equation (12) evaluates to

$$
\begin{aligned}
\int_{t=0}^{t} d t & =\frac{1}{\sqrt{A}} \int_{\theta_{0}}^{\kappa \sec \theta-\kappa} \frac{\kappa \sec \theta \tan \theta}{\sqrt{\kappa^{2} \sec ^{2} \theta-\kappa^{2}}} d \theta \\
& =\int_{\theta_{0}}^{\kappa \sec \theta-\kappa} \sec \theta d \theta \\
& =\frac{1}{\sqrt{A}} \ln \left|\frac{z+\kappa+\sqrt{(z+\kappa)^{2}-\kappa^{2}}}{\kappa}\right|_{z=0}^{z(t)} .
\end{aligned}
$$

Evaluated at its upper and lower limits, this equation gives us our position function of time, $z(t)$, which is a hyperbolic cosine function:

$$
z(t)=\kappa \cosh (t \sqrt{A})-\kappa
$$

The first-order approximation is valid as long as $\frac{z(t)}{r_{e}} \leq 0.1$, so the time domain $0 \leq t \leq T_{1}$ can be found as follows:

$$
T_{1}=\frac{1}{\sqrt{A}} \ln \left|\frac{0.1 r_{e}+\kappa+\sqrt{\left(0.1 r_{e}+\kappa\right)^{2}-\kappa^{2}}}{\kappa}\right|
$$

If equation (13) is plotted as a time function of position, then the reflection of the graph over $y=x$ will simply be the position function of time. Thus we have the first-order approximation 
of the rocket's first-stage position function with consideration of varying gravity and exponential fuel burn rate.

Of course, a rocket will almost never fly in a straight path governed by the equations above. Thus, models must be derived to account for other situations as well. Many rockets use the gravity turn technique in order to assume a stable orbit.

\section{GRAVITY TURN}

A rocket performing a gravity turn will fly in a rectilinear path orthogonally outward from the surface of the Earth until it reaches a predetermined displacement from its starting point. At that displacement, the rocket's aim is slightly shifted by a momentarily redirected thrust so the rocket is tilted at an angle to the vertical, which is defined as the axis perpendicular to the Earth's surface. The force of thrust from the rocket's nozzle then acts strictly along the axis parallel to the length of the rocket while gravity provides torque. This torque eventually causes the rocket to level out with the horizontal.

The goal of the maneuver is to prevent too much thrust from being used in the direction opposite to gravity and to let the rocket gain horizontal velocity while gaining vertical position. A rocket has to meet a certain threshold velocity and position before it can maintain itself in L.E.O. (low Earth orbit), so the ideal goal is to meet those markers before the rocket levels out with the horizontal.

We will be making the simplifying assumption that a rocket's gravity turn takes place over a nearly circular arc so that we may arrive at a solution made up of elementary functions, see Figure 1. 


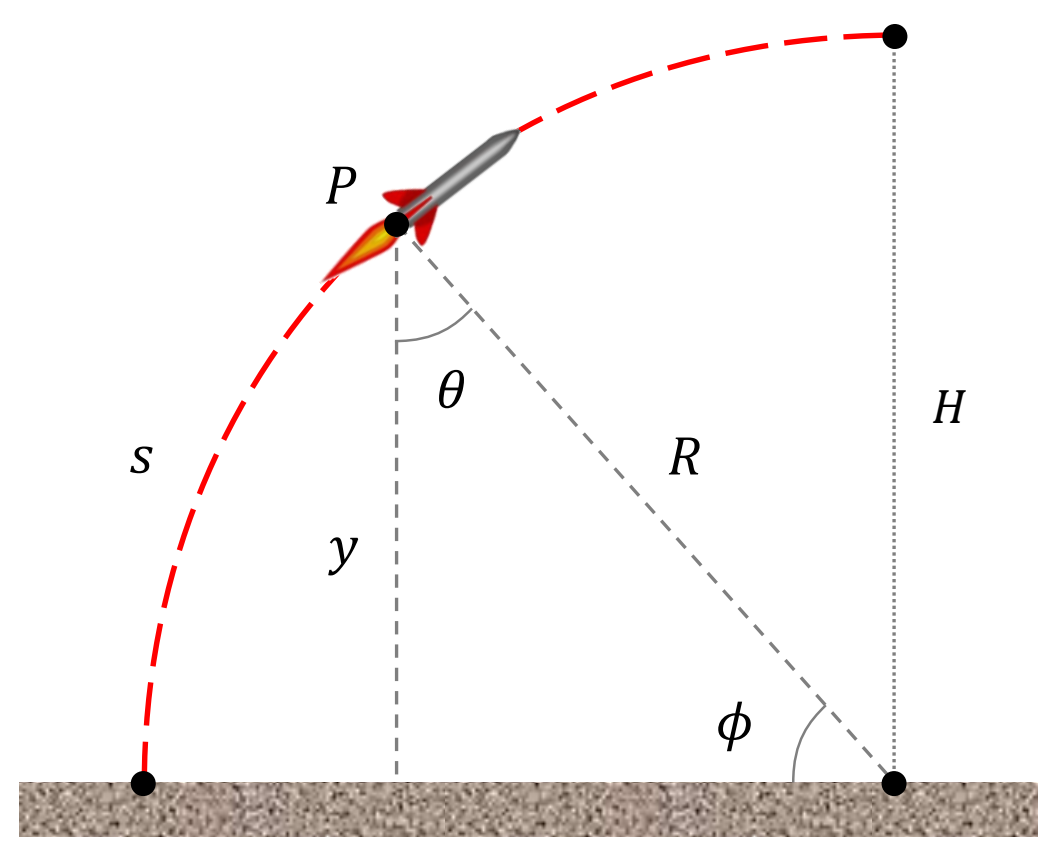

Figure 1: Rocket performing a gravity turn (not drawn to scale)

\section{CONSTANT GRAVITY ASSUMPTION}

The forces acting on a rocket undergoing a gravity turn include gravity, thrust, and drag. Assuming drag is negligible, we are left with gravity and thrust. If we assume that the force of gravity acting on the rocket is constant and equal to $m g$, then we can write a differential equation as follows:

$$
\sum_{i=1}^{n} F_{i}=-c \frac{d m}{d t}-m g \sin \theta=m \frac{d v}{d t}
$$

where the thrust term is negative because mass is decreasing and $\sin \theta$ is multiplied by $m g$ because $m g \sin \theta$ is the component of gravity acting opposite to thrust. It is now necessary to use our mass assumption to simplify equation (14). We arrive at

$$
\frac{d v}{d t} m_{0} e^{-Q t}=-m_{0} e^{-Q t} g \sin \theta+Q c m_{0} e^{-Q t}
$$


Simplifying,

$$
Q c-g \sin \theta=\frac{d v}{d t}=\frac{d v}{d s} \frac{d s}{d t}=v \frac{d v}{d s}
$$

which can be solved by determining a way to rewrite $\sin \theta$ in terms of $s$. A diagram relating the arc length $s$ covered by the rocket at an arbitrary point $P$ to $\theta$ is shown below.

This diagram relates all of the relevant distance quantities and angles that we will be dealing with, so it will be useful throughout the exploration. For now, it is important to notice that $\theta$ is a function of time and is approaching zero as the point $P$ approaches the top of the arc. At any point $P$,

$$
\sin \theta=\frac{\sqrt{R^{2}-y^{2}}}{R}
$$

where $\sqrt{R^{2}-y^{2}}$ is the side of the right triangle opposite to $\theta$ by Pythagoras' theorem. Using the circle-sector relationship, i.e., arclength $=$ radius $\times$ angle, we can write

$$
s=R \phi=R \sin ^{-1}\left(\frac{y}{R}\right) \quad \Rightarrow \quad y=R \sin \left(\frac{s}{R}\right)
$$

Combining equations (15), (16), and (17), we have

$$
v \frac{d v}{d s}=Q c-g \frac{\sqrt{R^{2}-R^{2} \sin ^{2}\left(\frac{s}{R}\right)}}{R}=Q c-g \frac{\sqrt{R^{2}\left[1-\sin ^{2}\left(\frac{s}{R}\right)\right]}}{R} .
$$

Using the trigonometric identity $\cos ^{2} \theta=1-\sin ^{2} \theta$, we can simplify this equation to

$$
v \frac{d v}{d s}=Q c-g \cos \left(\frac{s}{R}\right)
$$

We can integrate this equation as follows: 


$$
\int_{v_{0}}^{v(t)} v d v=\int_{s=0}^{s(t)}\left[Q c-g \cos \left(\frac{s}{R}\right)\right] d s \quad \Rightarrow \quad \frac{[v(t)]^{2}}{2}-\frac{v_{0}^{2}}{2}=Q c s(t)-g R \sin \left(\frac{s(t)}{R}\right) .
$$

This is another first order differential equation, but unlike the first, it is not solvable. In order to approximate solutions numerically, however, we must rearrange as if we were going to solve the equation. $v(t)$ must become $\frac{d s}{d t}$, and $s(t)$ must again become $s$ for the purpose of integration:

$$
\frac{d s}{d t}=\sqrt{v_{0}^{2}-2 g R \sin \left(\frac{S}{R}\right)+Q c s}
$$

which means that

$$
\int_{t=0}^{t} d t=\int_{s=0}^{s(t)} \frac{d s}{\sqrt{v_{0}^{2}-2 g R \sin \left(\frac{S}{R}\right)+Q c s}}
$$

Similar to (8), this particular integral does not have a closed form-solution made up of elementary functions. As such, we must deal with it numerically. Before doing so, however, let us analyze a slightly more accurate approximation — one which accounts for varying gravitational force.

\section{VARYING GRAVITY ASSUMPTION}

The distance over which a gravity turn takes place is usually much longer than the rest of the rocket flight. As such, modeling gravity by the inverse square law $\frac{k}{(h+y)^{2}}$, where $h$ is the height where the rocket begins the gravity turn and $k=m_{e} G$, rather than the static model $m g$ substantially impacts the projected trajectory of the rocket during the gravity turn. Luckily, the derivation of the model with the additional varying gravity assumption is largely similar to constant gravity model: 


$$
v \frac{d v}{d s}=-\frac{k}{(h+y)^{2}} \sin \theta+Q c
$$

In (17) we noted that $y=R \sin \left(\frac{s}{R}\right)$ and used this observation to show that $\sin \theta=\cos \left(\frac{s}{R}\right)$.

This means that $v \frac{d v}{d s}=-\frac{k \cos \left(\frac{s}{R}\right)}{\left(h+R \sin \left(\frac{s}{R}\right)\right)^{2}}+Q c$; separating variables and integrating as before yields

$$
\int_{v_{0}}^{v(t)} v d v=\int_{s=0}^{s(t)}\left[-\frac{k \cos \left(\frac{s}{R}\right)}{\left(h+R \sin \left(\frac{s}{R}\right)\right)^{2}}+Q c\right] d s \Rightarrow \frac{v(t)^{2}-v_{0}^{2}}{2}=\frac{k}{h+R \sin \left(\frac{s(t)}{R}\right)}-\frac{k}{h}+Q \operatorname{cs}(t) .
$$

Isolating $\frac{d s}{d t}$, separating variables, and integrating, we get

$$
\int_{t=0}^{t} d t=\int_{s=0}^{s(t)} \frac{d s}{\sqrt{v_{0}^{2}+\frac{2 k}{h+R \sin \left(\frac{s}{R}\right)}-\frac{2 k}{h}+2 Q c s}}
$$

\section{NUMERICAL APPROXIMATIONS}

We can test the accuracy of the constant gravity assumption by performing numerical approximations on both models (18) and (19), seeing how they differ. To do this, we will use the method of trapezoidal sums to approximate the integrals. The quantities we must know include the velocity and height of the rocket as it begins its turn and the goal height for the end of the maneuver.

\section{APPROXIMATION COMPARISONS}

A well-known height at which a rocket should start its gravity turn is $10 \mathrm{~km}$. To find the velocity with which the rocket starts its turn, we must determine the rocket's position and velocity functions of time before it enters the turn. This is relatively simple if we assume that gravity is constant, which we can do in this situation because the ratio of the gravitational force 
$F_{1}$ acting on an object of mass $m$ on Earth's surface to the gravity $F_{2}$ acting on the same object at $10 \mathrm{~km}$ is

$$
\frac{F_{1}}{F_{2}}=\frac{\frac{G m_{e} m}{r_{e}^{2}}}{\frac{G m_{e} m}{\left(r_{e}+10,000\right)^{2}}}=\frac{\left(r_{e}+10,000\right)^{2}}{r_{e}^{2}}=\frac{\left(6.38 \times 10^{6}+10,000\right)^{2}}{\left(6.38 \times 10^{6}\right)^{2}} \approx 1.003 .
$$

With proper significant figures, the ratio is approximately equal to 1.00 , so the difference between the two is negligible for our purposes. With that said, Newton's Second Law gives us

$$
m \frac{d v}{d t}=-m g-c \frac{d m}{d t} \Rightarrow \frac{d v}{d t}=Q c-g \Rightarrow \int_{v=0}^{v(t)} d v=\int_{t=0}^{t}(Q c-g) d t \Rightarrow v(t)=(Q c-g) t
$$

which means $\int_{z=0}^{z(t)} d z=(Q c-g) \int_{t=0}^{t} t d t$ and

$$
z(t)=\left(\frac{Q c-g}{2}\right) t^{2}
$$

Using data released by SpaceX from the first stage of a Falcon 9 launch, $Q=0.075 / \mathrm{s}$ and $c=2,840 \mathrm{~m} / \mathrm{s}$. Therefore, according to equation (9), at $z=10,000 \mathrm{~m}$,

$$
t=\sqrt{\frac{2(10,000)}{(0.075)(2,840)-9.81}} \approx 9.92 \text { seconds } .
$$

Substituting $t=9.92$ seconds into equation (8) gives $v(9.92) \approx 2,020 \mathrm{~m} / \mathrm{s}$.

Assuming $H=6,540,000 \mathrm{~m}$, which is the beginning of low-Earth orbit relative to Earth's center, the radius of curvature of the rocket's gravity turn is equal to

$$
R=H-h=6,540,000-\left(10,000+6.38 \times 10^{6}\right)=150,000 \mathrm{~m} .
$$


Substituting our numerical data into the gravity turn equation (6) which we derived under the assumption of constant gravity,

$$
t=\int_{s=0}^{s(t)} \frac{d s}{\sqrt{4,080,400-2943,000 \sin \left(\frac{s}{150,000}\right)+213 s}}
$$

where $t$ is equal to the time it takes for the rocket to travel from $s=0$ to $s(t)$. Once the rocket has traveled $\frac{150,000 \pi}{2} m$, it is horizontal (at the top of the arc). To test the accuracy of this function, we will perform a numerical approximation of the integral from $s=0$ to $S=\frac{150,000 \pi}{2}$ using trapezoidal sums of eight subdivisions.

The formula for the trapezoidal approximation with $n$ subdivisions of the integral of a function $f(x)$ is

$$
\int_{a}^{b} f(x) d x \approx \frac{b-a}{2 n}\left[f\left(x_{0}\right)+2 f\left(x_{1}\right)+2 f\left(x_{2}\right)+\ldots+2 f\left(x_{n-1}\right)+f\left(x_{n}\right)\right]
$$

where $f\left(x_{0}\right)=f(a)$ and $f\left(x_{n}\right)=f(b)$. Using this formula to approximate our integral with $n=8$, we arrive at

$$
t=\int_{s=0}^{75,000 \pi} \frac{d s}{\sqrt{4,080,400-2,943,000 \sin \left(\frac{s}{150,000}\right)+213 s}} \approx 52.5 \text { seconds. }
$$

Substituting the same numerical data into our more accurate integral equation and replacing $k$ with $G m_{e}=7.96 \times 10^{14}$, we arrive at

$$
t=\int_{s=0}^{s(t)} \frac{d s}{\sqrt{4,080,400+\frac{7.96 \times 10^{14}}{6,390,000+150,000 \sin \left(\frac{s}{150,000}\right)}-116,374,269+426 s}} \approx 37.2 \text { seconds. }
$$


As should be expected, the equation based on a varying gravitational force states that the rocket takes less time than it would under a constant gravitational force $\mathrm{mg}$. This is because gravity decreases as the rocket moves farther and farther from Earth, so the net force acting against it also decreases, making it easier for the rocket to reach its goal at low-Earth orbit. The percent error of the constant gravity assumption for this particular approximation (assuming that the varying gravity approximation is accurate) is $\frac{|37.2-52.5|}{37.2} \times 100=41.1 \%$. Clearly, assuming a constant gravitational force here is not going to yield accurate results.

\section{GRAPHICAL CONFIRMATION}

We can check the results of our numerical approximations by plotting the integrals as time functions of position like so:
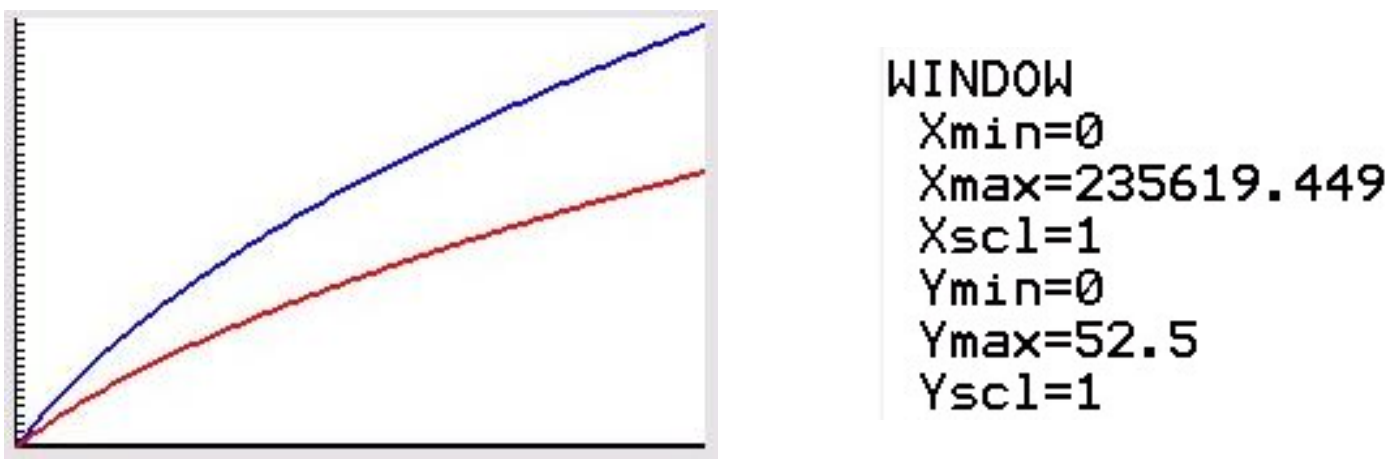

Figure 2: Comparison of a rocket trajectory on a gravitational turn with the assumption of constant gravity (blue) versus inverse square distance gravity (red).

The upper curve is the plot of the function derived from constant gravity assumptions, while the lower curve is the model based upon varying gravity. Both are plotted from $s=0$ to $s=$ $75000 \pi$, with the $y$-axis representing time and the $x$-axis representing position. The graph is an excellent visual display of the disparity between the constant gravity and varying gravity functions. To address the accuracy of the trapezoidal approximations, the constant gravity graph reads $t=51.8$ seconds at $s=75,000 \pi$, while the varying gravity graph reads $t=33.8$ 
seconds at the same $s$ position. In the constant gravity case, the error is less than $5 \%$, making the numerical approximation valuable for most applications. In the varying gravity case, however, error is closer to $10 \%$, meaning a higher n-value would need to be chosen for an accurate approximation with trapezoidal sums.

\section{VELOCITY IN ORBIT}

The final part of this exploration focuses on the velocity necessary to maintain a rocket in low-Earth orbit. When an object is in orbit, it is actually falling around the Earth fast enough such that it doesn't appear to be falling at all. This is why a rocket must reach a threshold velocity before it can stay in orbit. In circular motion, an object's centripetal acceleration (the acceleration vector perpendicular to the object's velocity vector) is $a_{c}=\frac{v^{2}}{r}$, where $r$ is the distance from the center of the circle to the object. Thus for an object of mass $m$ in circular motion $\sum_{i=1}^{n} F_{i}=\frac{m v^{2}}{r}$. Once the rocket reaches orbit at height $H$, it experiences only the force of gravity in the direction of centripetal acceleration, i.e., $\frac{m v_{h}^{2}}{H}=\frac{k m}{H^{2}} \Rightarrow v_{h}=\sqrt{\frac{k}{H}}$. When $H=\left(160+r_{e}\right) \mathrm{km}$, it follows that $v_{h}=\sqrt{\frac{7.96 \times 10^{14}}{160,000+6.38 \times 10^{6}}} \approx 11.0 \mathrm{~km} / \mathrm{s}$.

Now we must determine whether the rocket with our specifications will meet the threshold velocity at this particular orbit. To do this, we will use the velocity function of position derived earlier but restated here:

$$
\frac{v(s(t))^{2}-v_{0}^{2}}{2}=\frac{k}{h+R \sin \left(\frac{s(t)}{R}\right)}-\frac{k}{h}+Q \operatorname{cs}(t) .
$$

Solving for $v(s(t))$ when $s(t)=75,000 \pi$, gives $v(75,000 \pi) \approx 10.1 \mathrm{~km} / \mathrm{s}$. 


\section{DISCUSSION}

At this point, we must consider the reasons why our rocket did not meet the threshold. The first observation to make is that this is actually the threshold velocity for the lowest point that is still considered low-Earth orbit. This means that if the rocket with the same specifications had tried to reach a higher orbit, it would have probably met that orbit's threshold velocity. The formula derived above for the required velocity shows that it decreases as the distance from the center of orbit increases. Also, the rocket would have been given more time to accelerate if it had aimed for a higher orbit. The second observation to make is that this example shows the importance of effective exhaust velocity. The higher the effective exhaust velocity of a rocket, the easier it will be for that rocket to meet the required velocity for orbit.

\section{CONCLUSION AND RECOMMENDATIONS}

The ideal goal is to choose an exhaust speed, relative to the rocket, that will not accelerate the rocket too far past the goal velocity. If the rocket does accelerate past the goal velocity, energy, and thus money, has been wasted. Finally, the specs used to examine our derived formulae are from one of the Falcon 9 flights. We did not consider the many possible variations SpaceX employed on its Falcon 9 launches. This particular launch, for example, was a staged rocket.

This model is being extended through the inclusion of the effects of friction. Thus far, friction has made the problem quite a bit more challenging, for a realistic friction model (for a fast moving rocket) would see drag to be dependent on the square of the rocket's velocity. 


\section{NOMENCLATURE}

$r_{e} \quad$ Earth's radius, or $6.38 \times 10^{6} \mathrm{~m}$

G Newton's gravitational constant

$g \quad$ Acceleration due to gravity at Earth's surface, or $9.81 \mathrm{~m} / \mathrm{s}^{2}$

$m_{e} \quad$ Earth's mass, or $5.97 \times 10^{24} \mathrm{~kg}$

$m_{0} \quad$ Initial mass of the rocket at time $t=0$

$m \quad$ Rocket's mass (varies with time)

$Q \quad$ Proportionality constant relating $m(t)$ to its derivative $\left(s^{-1}\right)$

c Exhaust speed relative to the rocket

$v \quad$ Speed of the rocket relative to the Earth's center (varies with time)

$v_{0} \quad$ Initial speed of the rocket at time $t=0$

$y \quad$ Position of the rocket relative to the Earth's center (varies with time)

$y_{0} \quad$ Initial position of the rocket at time $t=0$

$h \quad$ Height when the rocket begins the gravity turn

$H \quad$ Goal height for the second stage

$z \quad$ Position of the rocket relative to the Earth's surface (varies with time)

$\alpha \quad$ Constant equal to $G m_{e}$

$\beta \quad$ Constant equal to $Q c$

$A \quad$ Constant equal to $\frac{2 g}{r_{e}}$

$B \quad$ Constant equal to $2 \beta-2 g$

$\kappa \quad$ Constant equal to $\frac{B}{2 A}$ 


\section{REFERENCES}

Betts, John. "Survey of Numerical Methods for Trajectory Optimization." Journal of Guidance, Control, and Dynamics 21.2 (1998): 193-207.

Coburn, Nathaniel. "Optimum Rocket Trajectories." Technical Report. University of Michigan, 1950. <http://hdl.handle.net/2027.42/4306>.

Hoburg, Warren. Aircraft Design Optimization as a Geometric Program. PhD Thesis. Berkeley: University of Califorinia, 2013.

Rankin, RA. "The Mathematical Theory of the Motion of Rotated and Unrotated Rockets." Philosophical Transactions A 241.837 (1949): 457-584.

Sutton, George P and Oscar Biblarz. Rocket Propulsion Elements. 8th Edition. John Wiley \& Sons, 2010.

Tawakley, VB. "Recent Developments in Rocket Flight Optimization Problems." Defence Science Journal 16.4A (1966): 91-98. 